\title{
Quantifying Protein-Ligand Binding Constants using Electrospray lonization Mass Spectrometry: A Systematic Binding Affinity Study of a Series of Hydrophobically Modified Trypsin Inhibitors
}

\author{
Dragana Cubrilovic, ${ }^{1}$ Adam Biela, ${ }^{2}$ Frank Sielaff, ${ }^{2}$ Torsten Steinmetzer, ${ }^{2}$ Gerhard Klebe,${ }^{2}$ \\ Renato Zenobi ${ }^{1}$ \\ ${ }^{1}$ Department of Chemistry and Applied Biosciences, ETH Zurich, Zurich, Switzerland \\ ${ }^{2}$ Department of Pharmaceutical Chemistry, Phillipps University Marburg, Marburg, Germany
}

\begin{abstract}
NanoESI-MS is used for determining binding strengths of trypsin in complex with two different series of five congeneric inhibitors, whose binding affinity in solution depends on the size of the P3 substituent. The ligands of the first series contain a 4-amidinobenzylamide as P1 residue, and form a tight complex with trypsin. The inhibitors of the second series have a 2-aminomethyl5-chloro-benzylamide as P1 group, and represent a model system for weak binders. The five different inhibitors of each group are based on the same scaffold and differ only in the length of the hydrophobic side chain of their P3 residue, which modulates the interactions in the S3/4 binding pocket of trypsin. The dissociation constants $\left(K_{D}\right)$ for high affinity ligands investigated by nanoESI-MS ranges from $15 \mathrm{nM}$ to $450 \mathrm{nM}$ and decreases with larger hydrophobic P3 side chains. Collision-induced dissociation (CID) experiments of five trypsin and benzamidine-based complexes show a correlation between trends in $\mathrm{K}_{\mathrm{D}}$ and gas-phase stability. For the second inhibitor series we could show that the effect of imidazole, a small stabilizing additive, can avoid the dissociation of the complex ions and as a result increases the relative abundance of weakly bound complexes. Here the $K_{D}$ values ranging from 2.9 to $17.6 \mu \mathrm{M}$, some $1-2$ orders of magnitude lower than the first series. For both ligand series, the dissociation constants $\left(K_{D}\right)$ measured via nanoESI-MS were compared with kinetic inhibition constants $\left(\mathrm{K}_{\mathrm{i}}\right)$ in solution.
\end{abstract}

Key words: Noncovalent interactions, Electrospray ionization mass spectrometry, Binding affinity, Trypsin, Protein-ligand complexes, Hydrophobic effect

\section{Introduction}

Q lectrospray ionization mass spectrometry (ESI-MS) is a Dowerful and increasingly utilized tool for the investi-

Electronic supplementary material The online version of this article (doi:10.1007/s13361-012-0451-6) contains supplementary material, which is available to authorized users.

Correspondence to: Renato Zenobi; e-mail: zenobi@org.chem.ethz.ch gation of noncovalent interactions [1]. This soft ionization technique allows the transfer of noncovalent complexes from solution into the gas phase and their subsequent study by mass spectrometry. To this day, protein-protein, protein-small molecule, protein-DNA, and DNA-small molecule complexes have been successfully detected and studied by this method [2-4]. Especially in drug discovery ESI-MS is of increasing importance for the investigation of protein-ligand interactions and determination of binding affinities [5, 6]. In recent years, binding affinities $\left(\mathrm{K}_{\mathrm{D}}\right)$ have been successfully determined by 
ESI-MS for a variety of noncovalent protein-ligand complexes $[2,7,8]$. Other MS-based methods have also been successfully applied for quantifying interactions (e.g., methods dubbed "protein-ligand interactions in solution by MS, titration and $\mathrm{H} / \mathrm{D}$ exchange" (PLIMSTEX) and "stability of unpurified proteins from rates of H/D exchange" (SUPREX) [9-11].

Noncovalent interactions are of great importance in nature; for example they play a major role in stabilizing protein conformation. The hydrophobic effect plays an important role in protein folding, in the adhesion of lipid bilayers, nucleic acid structures, and protein-small molecule interactions [12-14]. Compared with the aqueous environment, the hydration shell is absent in the gas phase, and its not yet fully clear whether the conformation of noncovalent complexes remains unchanged during the transition from solution to vacuum [15]. Some forces such as hydrogen-bonding and electrostatic interactions between two oppositely charged molecules are strengthened in the gas phase, while hydrophobic interactions are weakened and therefore difficult to preserve during ionization and ion transfer $[16,17]$. A number of research groups have reported investigations of noncovalent complexes where hydrophobic interactions play a dominant role for the complex stability [1820]. In a very recent study, Klassen and co-workers [21, 22] demonstrated the application of ESI-MS to quantify binding strengths of $\beta$-lactoglobulin - fatty acid complexes in aqueous solution. For three short fatty acids, association constants smaller than expected were found by the authors $\left(\mathrm{K}_{\mathrm{a}}\right.$ compared with data from a competitive fluorescence assay). They explained this with an in-source dissociation, which reduces the relative abundance of gaseous complex ions measured by ESI-MS. In a previous study of the same research group it was shown that $\beta$-lactoglobulin retains the structure of its binding cavity even in the absence of a hydration shell [23]. The authors monitored the dissociation of the fatty acid-protein complexes in a BIRD experiment and extracted the temperature-dependent kinetic parameters. Their results show that the energy required for dissociation correlates with the length of the hydrocarbon fatty acid chain. Surprisingly, quantitative comparison of the dissociation rate constants in the hydrated and dehydrated states showed that the solvated complex is kinetically less stable than the corresponding gaseous ions at all temperature investigated [24].

Even when carefully controlling the instrument parameters, complexes that are predominatly stabilized by nonpolar interactions are prone to dissociation in the gas phase [25]. This so-called in-source dissociation can lead to an artificially low binding constants based on the reduced abundance of the complex ions [21]. Stabilization by addition of imidazole to the nanoES solution was presented as a solution for this problem for several weakly bound complexes [26, 27]. The small imidazole molecule acts as a nonspecific, sacrificial ligand and can prevent dissociation of the specifically bound ligand during ES-MS analysis. This can also be thought of as enhanced evaporative cooling of the protein-ligand complex ions in the ion source. The extent of the stabilization depends strongly on the concentration of imidazole. At high imidazole concen- trations $(>1 \mathrm{mM})$, the ions of protein-fatty acid, proteincarbohydrate and protein-small molecule complexes can be stabilized [26], although it is not always possible to prevent dissociation of very labile gas-phase complexes, [26, 28, 29].

To determine binding constants, we used the ES-MS titration method, which has been found suitable for measuring binding strengths, not only in our laboratory but also in other groups [2, 18, 30-32]. It has also been validated against more established biophysical methods, such as isothermal titration calorimetry (ITC), surface plasmon resonance (SPR), and nuclear magnetic resonance (NMR) spectroscopy [32-36]. The well-described titration method relies on detection of ions belonging to the complex versus bare proteins. The $K_{D}$ value can then be easily determined from a fit of the intensity ratio of bound and unbound protein as a function of the added ligand. This method assumes that no dissociation takes place during the transmission through the mass spectrometer. The second important assumption is that the intensity ratio observed in the gas phase correlates with the concentration ratio in solution. If a very low-mass ligand is bound to a high-mass protein, the ionization efficiency does not change for the complex vs. the bare protein, and this assumption is fulfilled in almost all cases [2]. As the ESI titration measurements can deliver a "snapshot" of the solution concentrations the $\mathrm{K}_{\mathrm{D}}$ values determined via nanoESI-MS reflect solution-phase binding affinities.

In this work, we present a systematic nanoESI-MS study for quantifying the binding strengths of trypsin in complex with two different series of competitive inhibitor. Closely related 4amidinobenzylamide- (AMBA) and 2-aminomethyl-5-chlorbenzylamide-based (CMA) inhibitors were chosen to gauge how their hydrophobic side chains modulate the interaction (Table 1). The two different types of inhibitors are based on the same scaffold and each series varies only in the length of the hydrophobic P3 side chain. In essence, we expected that the binding affinity should increase with increasing length of the hydrophobic chain that is accommodated by the predominantly hydrophobic S3/4 cavity of the enzyme. We are using two compound model systems: benzamidine-type ligands that exhibit strong binding affinity to trypsin, while the CMAbased ligands show lower. The protein used in this study, bovine $\alpha$-trypsin, belongs to the family of the well-known serine proteases. Compounds that specifically inhibit these serine proteases can be used for the treatment of different diseases. For example, in the last years the first orally available thrombin and factor Xa inhibitors were approved as anticoagulants $[35,36]$.

The basic question is whether we can observe the expected trend in the binding affinity of the inhibitors, when using ESIMS as a read-out for the solution phase equilibrium. In the case of the benzamidine-based ligands, the binding affinity based on ESI titration measurements increases with increasing length of the hydrophobic P3 side chain, from 15 to $450 \mathrm{nM}$. A second question concerns the stability in solution vs. in the gas phase. In some studies it was shown that the gas-phase stability reflects the binding properties in solution [37-39]. More frequently, however, a correlation between the gas-phase 


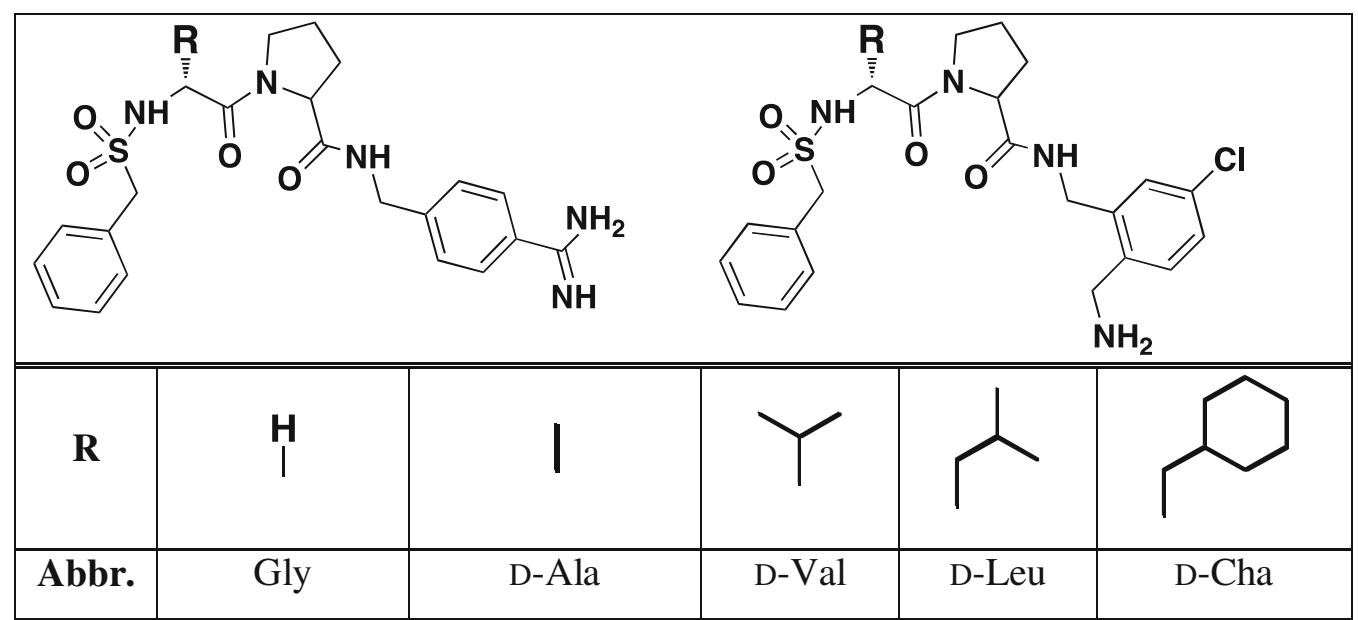

Table 1. Chemical Structures of the Scaffold of the Investigated Benzamidine- and CMA-type Ligands. The Residues (R) Indicate the Substructure of the Inhibitor, which Binds into the Hydrophobic S3/4 Pocket of Trypsin. This Part of the Ligand was Systematically Varied in the Size of the Hydrophobic Chain

stability and the solution-phase stability is absent $[25,26,28$, 40-46], for example for leucine-zippers and acyl-CoA binding protein (ACBP) and a series of acyl CoA derivatives [28]. If binding properties in solution correlate with gas-phase stability it has to be assumed that the dominant interactions are very similar in solution and in the gas phase, and that solvent mediation play only a minor role (see Daniel at al. and Sharon and Robinson for comprehensive reviews on this topic $[2,46])$. In our study the CID experiments for the benzamidine complexes show a correlation between the binding affinities in solution and the gas phase stability.

The complexes with the CMA inhibitors were prone to in-source dissociation. The main reason may be that complexes stabilized in solution by weak nonpolar interactions exhibit low gas-phase stability [27]. Therefore we have investigated the stabilizing effect of imidazole on this particular model system. Like Klassen and co-workers [21, 22] we found that the addition of imidazole to the nanoESI solution can protect protein-ligand complexes from in-source dissociation during the ESI-MS analysis process. Unlike in the case of the benzamidine series, the trend of higher binding affinity is clearly observed, with the order Gly $<$ DAla $<$ D-Leu. No independently measured $K_{D} S$ are available for these systems. Therefore, binding affinities determined by MS were compared with inhibition constants $\left(\mathrm{K}_{\mathrm{i}}\right)$ determined via an enzyme kinetic inhibition assay.

\section{Experimental}

\section{Materials and Methods}

Bovine pancreas $\alpha$-trypsin $(\mathrm{MW} \approx 23.300 \mathrm{Da})$, ammonium acetate, and CsI were purchased from Sigma Aldrich (Buchs, Switzerland). Imidazole (99.5 \% purity) and DMSO were obtained from Fluka Chemie AG. Water was purified using a Milli-Q Ultrapure water purification system by Millipore (Barnstead, IA / USA). All MS titration experiments were recorded under "native-like" conditions using
$50 \mathrm{mM}$ ammonium acetate buffer at $\mathrm{pH}=7.8$. Stock solutions of ligands were prepared at $40-50 \mathrm{mM}$ concentration in DMSO. Prior to the measurement, the inhibitor solutions were diluted with Milli-Q water to the desired concentration. The protein working solution was made from a $100 \mu \mathrm{M}$ stock solution in ammonium acetate buffer. The exact trypsin concentration was determined using a UV spectrometer (NanoDrop 1000; Witec AG, Littau, Switzerland).

\section{Mass Spectrometry}

ESI spectra were acquired with a hybrid quadrupole time-offlight mass spectrometer (Q-TOF ULTIMA; Waters/Micromass, Manchester, UK) in the positive ion mode. The instrument was controlled via the MassLynx ver. 4.0 software. In order to obtain a good signal-to-noise ratio, 100 scans were accumulated for one spectrum. The mass spectrometer is equipped with an automated chip-based nanoESI system (Nanomate 100; Advion Biosciences, Ithaca, NY, USA). It has a 96-well sample plate, a rack of 96 disposable, conductive pipet tips, and a nanospray chip containing $20 \times 20$ nozzles of $5 \mu \mathrm{m}$ diameter. For investigation of noncovalent complexes, appropriate instrumental conditions have to be found. In this case the desolvation must be sufficiently complete in order to get narrow peaks for the detected species but not to dissociate the noncovalent complex. This can take an influence on peak broadening because of the adduct formation with salt and buffer molecules from the spray solution. The settings described below were found to be a good compromise between the intact complex detection and sufficient desolvation of analytes. For all nanoESI-MS measurements the voltage was set to $1.8-1.9 \mathrm{kV}$ and a gentle backing pressure of 5 bar on the spray tip was used to assist the liquid sample flow. The source temperature was kept at $21{ }^{\circ} \mathrm{C}$. To prevent dissociation of the noncovalent complexes, the mass spectrometer was run with gentle desolvation parameters. The cone and first ion tunnel RF1 voltages, the parameters 
that control the kinetic energy of the ions in the source region of the mass spectrometer, were optimized to $40 \mathrm{~V}$ and $35 \mathrm{~V}$. After this stage, the ion beam passed a hexapole collision cell filled with argon (purity 5.0; PanGas). Collision-induced dissociation (CID) used in MS/MS experiments were preformed by adjusting the acceleration collision energy (CE) voltage until full dissociation of the parent complex ions was achieved. Calibration of the mass spectrometry instrument was performed using CsI clusters. The concentration of CsI was $2 \mu \mathrm{g} / \mathrm{uL}$ dissolved in water/2propanol $(1 / 1, \mathrm{vol} / \mathrm{vol})$.

\section{Data Processing}

Before data processing, each mass spectrum was smoothed (Savitzky-Gollay smooth) with the MassLynx 4.0 software (Waters, UK). For the $K_{D}$ determination the measured relative peak height intensity (I) ratios $(\mathrm{R})$ of the ligandbound protein $(\mathrm{P} \cdot \mathrm{L})$ to bare protein $(\mathrm{P}), R=I(P \cdot L) / I(P)$, were calculated for each spectrum. For this calculation, all charge states were taken into account. The experimentally calculated R-values were plotted versus the total inhibitor concentration. The equation proposed by Daniel et al. [2] was used to determine the dissociation constant $\left(\mathrm{K}_{\mathrm{D}}\right)$ from the titration curve fit:

$$
\frac{I(P \cdot L)}{I(P)}=\frac{1}{2}\left(-1-\frac{[P]_{0}}{K_{D}}+\frac{[L]_{0}}{K_{D}}+\sqrt{4 \frac{[L]_{0}}{K_{D}}+\left(\frac{[L]_{0}}{K_{D}}-\frac{[P]_{0}}{K_{D}}-1\right)}\right)
$$

It was assumed here that the ionization efficiency for the bare protein and the complex is equal, which allowed us to use the intensity ratios of free protein over complex instead of their concentrations in solution. The $\mathrm{K}_{\mathrm{D}}$ calculations and the fitting of the titration curves were performed using MATLAB software (2010a, The MathWorks, Natick, MA, USA).

\section{Kinetic Inhibition Assay}

Kinetic inhibition of bovine trypsin was determined photometrically at $405 \mathrm{~nm}$ using the chromogenic substrate Pefachrom tPa (LoxoGmbH, Dossenheim, Germany) according to the protocols described by Stürzebecher et al. [47] under the following conditions: $50 \mathrm{mM}$ Tris/ $\mathrm{HCl}(\mathrm{pH} 8.0), 154 \mathrm{mM}$ $\mathrm{NaCl}, 5 \%$ DMSO, and $0.1 \%$ polyethylene glycol (PEG) 8000 at $25{ }^{\circ} \mathrm{C}$ using different concentrations of substrate and inhibitor. $\mathrm{K}_{\mathrm{i}}$ values were determined at least in triplicate.

\section{Results and Discussion}

$K_{D}$-Determination of the Benzamidine-Based Inhibitors by the nanoESI-MS Titration Method

The benzamidine-based inhibitors in complex with trypsin represent a model system for the quantification of "high- affinity ligands." Figure 1a provides an example of a nanoESI mass spectrum for a solution of $5 \mu \mathrm{M}$ trypsin in the presence of D-Cha in $50 \mathrm{mM}$ aqueous ammonium acetate solution under nondenaturating conditions $(\mathrm{pH}=7.8)$. The narrow charge state distribution, predominantly $7+, 8+, 9+$, is characteristic for native conditions, whereby $8+$ is the most intense signal. In order to determine the dissociation constant via the titration method, a series of nanoESI experiments were performed with increasing inhibitor concentrations ranging from 0.5 to $5 \mu \mathrm{M}$. Figure $1 \mathrm{a}-\mathrm{d}$ shows representative mass spectra obtained for the noncovalent trypsin-D-Cha-inhibitor complex using four different ligand concentrations. As expected, the ratio of the complex to free protein ion signals increases with higher total inhibitor concentration. Already at $5 \mu \mathrm{M}$ inhibitor concentration, full complexation is reached because the benzamidine group binds strongly and specifically to the active site of the enzyme and occupies the S1 pocket of trypsin. Also, the amidinium group of benzamidine is a mimic of the guanidinium side chain of arginine. The stabilization of the benzamidine moiety in the S1 pocket of trypsin is based on the a bridge linking the positively charged amidinium group and the carboxylate group of Asp189 [35]. Three other hydrogen bonds to Ser190, Gly210, and a conserved water

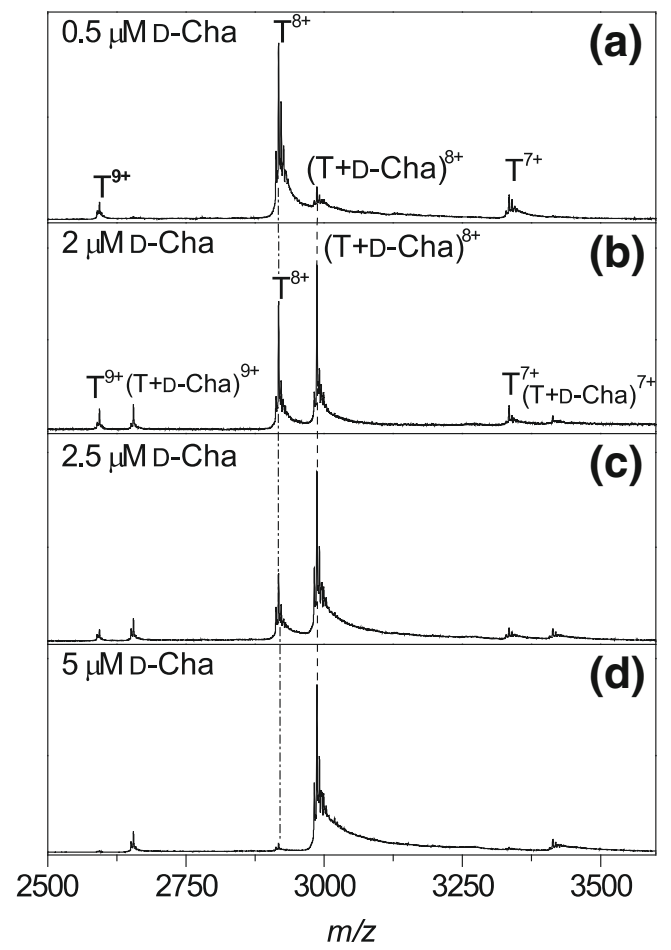

Figure 1. Representative nanoESI mass spectra of $5 \mu \mathrm{M}$ trypsin in the presence of the D-Cha-inhibitor obtained in positive ion mode under "native" conditions. Trypsin $(T)$ was titrated with different concentrations of the D-Cha inhibitor, (a) $0.5 \mu \mathrm{M}$, (b) $2 \mu \mathrm{M}$, (c) $2.5 \mu \mathrm{M}$, and (d) $5 \mu \mathrm{M}$. The signal for the noncovalent complex clearly increases with increasing amount of ligand present in solution. The full complexion is reached at $5 \mu \mathrm{M}$ inhibitor concentration 
molecule contribute to the strong ligand binding [35, 48, 49]. Moreover, additional van der Waals contacts between the $\mathrm{P} 1$ phenyl ring and residues of the $\mathrm{S} 1$ pocket of trypsin enhance the stability of the complex. The proline moiety of the inhibitor occupies the S2 pocket [50] (Table 1).

Once the optimized conditions were found for the D-Chainhibitor/trypsin complex, the protein was titrated against four other inhibitors. Spectra obtained with trypsin and equal concentrations $(2.5 \mu \mathrm{M})$ of three different inhibitors (Gly, D$\mathrm{Val}$, and D-Cha) are shown as examples in Figure 2. Based on the length of the hydrophobic side chain, D-Cha should show the strongest binding followed by D-Val and Gly. In other words, at the same inhibitor concentration the highest complex to the free protein ratio should be observed for D-Cha. The ratio between D-Cha-trypsin and free protein ions was 2.6, while D$\mathrm{Val}$ and Gly-trypsin generated ratios of 1.6 and 0.9 . The trend in increased binding affinity of these three inhibitors expressed by R-values is clearly confirmed by the spectra. These different hydrophobically modified residues bind into the hydrophobic S3/4 pocket of trypsin and are responsible for the observed increasing affinity. This pocket is supposed to favor binding of ligands possessing bulky aromatic moieties [51].

The stabilizing effect of imidazole, explained in detail below, was also tested on this particular model system. To test the influence of imidazole, a small stabilizing solution additive, we

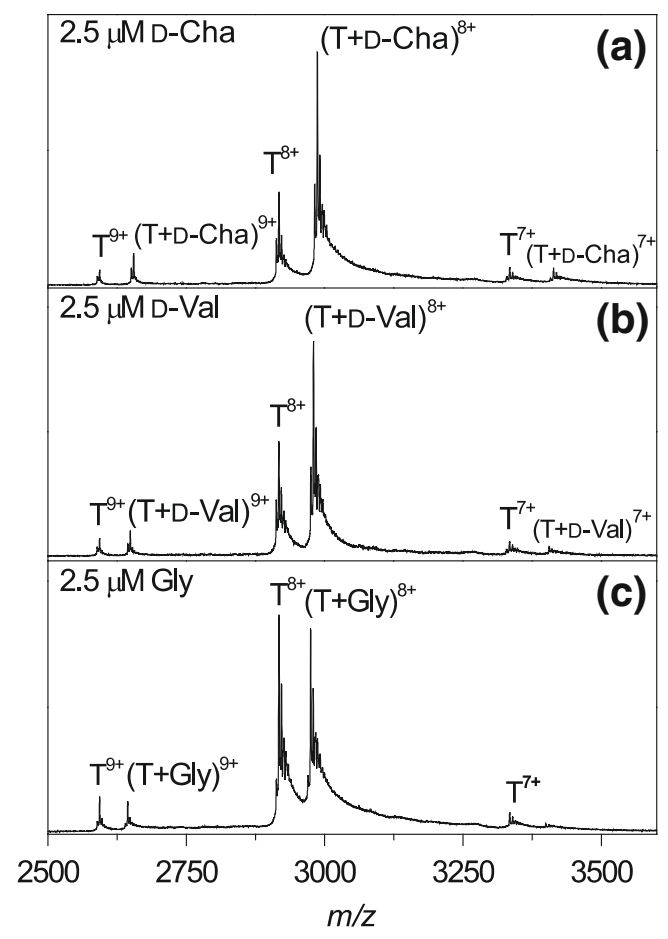

Figure 2. Representative nanoESI-MS spectra for three different complexes at the same inhibitor concentration $(2.5 \mu \mathrm{M})$. The ratio of the complex over the free protein expressed in $R$-values are (a) $D$-Cha $R=2.6$; (b) $D-V a l ~ R=1.6$; (c) Gly R=0.9 and confirmed the binding affinity order Gly < $\mathrm{D}-\mathrm{Val}<\mathrm{D}-\mathrm{Cha}$ by the spectra chose the D-Val-inhibitor. However, upon addition of $10 \mathrm{mM}$ imidazole to the nanoES solution, no change of the complex ions to the free protein was observed (data not shown). The only observable effect of imidazole was a shift of the charge state distribution to lower charge states. Since the ESI titration measurements were performed under "soft" sampling conditions and the benzamidine inhibitors bind specifically into all trypsin pockets, we assume that complexes are stable and not prone to dissociation in the gas phase.

The dissociation constants $\mathrm{K}_{\mathrm{D}}$ for all trypsin-inhibitor complexes were determined as described in the "Data Evaluation" section. Five different titration curves for the benzamidine-based inhibitors in complex with trypsin are shown in Figure 3. As can be seen on the curves a larger slope reflects a higher binding affinity and vice versa. The $\mathrm{K}_{\mathrm{D}}$-values based on ESI measurements and the $\mathrm{K}_{\mathrm{i}}$-values measured for five different inhibitors are compared in Table $2 . K_{D}$ s for binding with trypsin were in the range of $15 \mathrm{nM}$ to $449 \mathrm{nM}$. The largest jump in $\mathrm{K}_{\mathrm{D}}$ value was observed going from 79 to $15 \mathrm{nM}$, when the hydrophobic P3 side chain binding in the $S 3 / 4$ pocket increased in size from a D-Leu group to D-Cha. The comparison with inhibition constants $\left(\mathrm{K}_{\mathrm{i}} \mathrm{s}\right)$ was not to validate the $\mathrm{K}_{\mathrm{D}}$ values, but to see whether they follow a similar trend as that found for the $\mathrm{K}_{\mathrm{i}}$ values; depending on assay conditions, $\mathrm{K}_{\mathrm{D}} \mathrm{S}$ and $\mathrm{K}_{\mathrm{i}} \mathrm{s}$ need not be identical. It should also be noted that most of the $\mathrm{K}_{\mathrm{i}} \mathrm{s}$ for thrombin are smaller than those for trypsin. The reason is the known preference of thrombin for inhibitors with proline as a P2 residue, which fits perfectly below the thrombin specific 60-insertion loop making strong hydrophobic contacts to residues Tyr60A and Trp60D. The $\mathrm{K}_{\mathrm{i}}$ values for trypsin determined via the kinetic inhibition assay increased with larger hydrophobic side chain with exception of D-Val (Table 2). However, the kinetic inhibition constants for D-Cha, D-Leu and D-Val are very similar and in the low nM-range.

The discrepancy between $\mathrm{K}_{\mathrm{D}}$ and $\mathrm{K}_{\mathrm{i}}$ values can be easily explained by different assay conditions: for the MS-based $\mathrm{K}_{\mathrm{D}}$ determination, ammonium acetate was used as a buffer, while for the kinetic inhibition assay, a buffer solution of $50 \mathrm{mM}$ Tris/ $\mathrm{HCl}(\mathrm{pH} 8.0), 154 \mathrm{mM} \mathrm{NaCl}$, $5 \%$ DMSO, and $0.1 \%$ polyethylene glycol (PEG) was used. Different buffer solutions may have a substantial influence on the binding affinities for benzamidine-trypsin complexes. Since benzamidine inhibitors are very potent, the $\mathrm{K}_{\mathrm{i}}$ determination using chromogenic substrate are close to the instrument limit of detection. The consequence is that even marginal measurement inaccuracies would have significant influence on the $\mathrm{K}_{\mathrm{i}}$ values. This might be a possible explanation for observing higher binding affinity for D-Val instead of D-Cha. However, with the exception of $\mathrm{D}-\mathrm{Val}$, the $\mathrm{K}_{\mathrm{D}} \mathrm{S}$ and the $\mathrm{K}_{\mathrm{i}}$ values that were determined show that relative binding affinities of the different inhibitors can be successfully determined, and that the $\mathrm{K}_{\mathrm{D}}$ and $\mathrm{K}_{\mathrm{i}}$ values decrease with larger hydrophobic side chain. 
Figure 3. NanoESI-MS titration curves for the binding of five different inhibitors to trypsin. The ligand concentration ranges from 0.5 to $5 \mu \mathrm{M}$, while the protein concentration was kept constant. This titration method relies on the relative abundance of bare protein and complex ions measured by nanoESI-MS assuming that the intensity ratio observed in the gas phase correlates with the concentration ratio corresponding to the free protein and complex concentration in solution

\section{Gas Phase Stability versus Binding Affinity}

Collision-induced dissociation (CID) experiments were performed to gain additional information about the stability of different noncovalent trypsin-inhibitor complexes in the gas phase. For the MS/MS measurements the $8^{+}$complex ions were selected and dissociated during the transmission through the mass spectrometer. The collision energy offset was varied until the selected parent ions of different noncovalent complexes were completely dissociated. Figure 4 shows MS/MS spectra of three different Gly-, D-Leu-, D-Cha- ligand-trypsin complexes with a collision energy offset of $20 \mathrm{~V}$. Dissociation of the precursor ions (complex) yielded the $\mathrm{P}^{7+}$ and $\mathrm{P}^{8+}$ ions. The extent of dissociation of three different complexes at the same collision energy offset is obvious in the spectra: $53 \%$ of the D-Cha-inhibitor/ trypsin-complexes remained intact, while of the D-Leu and Gly complexes $45 \%$ and $34 \%$ survive, respectively. Since the increase in binding affinity of the different benzamidine inhibitors is based on the differences in their hydrophobic P3 side chain, a correlation between the solution-phase binding affinity and the gas-phase stability for D-Cha, D-Leu, and Gly complexes could be established by a single measurement.

Several groups have tried to find a correlation between the gas-phase stability and the type of interactions involved in a complex. As already mentioned in the Introduction, it is important to note that the gas-phase stabilities of noncovalent complexes generally do not correlate with solution binding affinities.

In our work, $\mathrm{CE}_{50}$ values, the collision energy offset where $50 \%$ of the complexes are dissociated, were used as a measure for the gas-phase stability of the complex. The dissociation curves for the different noncovalent complexes are shown in Figure 5, where the normalized percentage of the intact complex $100 \times I_{(T+L)} /\left(I_{(T+L)}+I_{T}\right)$ was plotted against the collision energy offset. $\mathrm{CE}_{50}$-values for the five different complexes reveal different dissociation rates during their transmission through the mass spectrometer. While $50 \%$ of the D-Cha complex dissociates at a collision energy setting of only $21 \mathrm{~V}$, only $16 \%$ of the Gly complex stays intact at the same collision energy. The data indicate that a correlation between the gas phase stability and the binding affinity in solution exists, showing a general trend towards higher stability with increasing hydrophobic P3 side chain. This correlation between solution-phase and gas-phase stability of the complexes suggests that polar and/or electrostatic contacts domi-

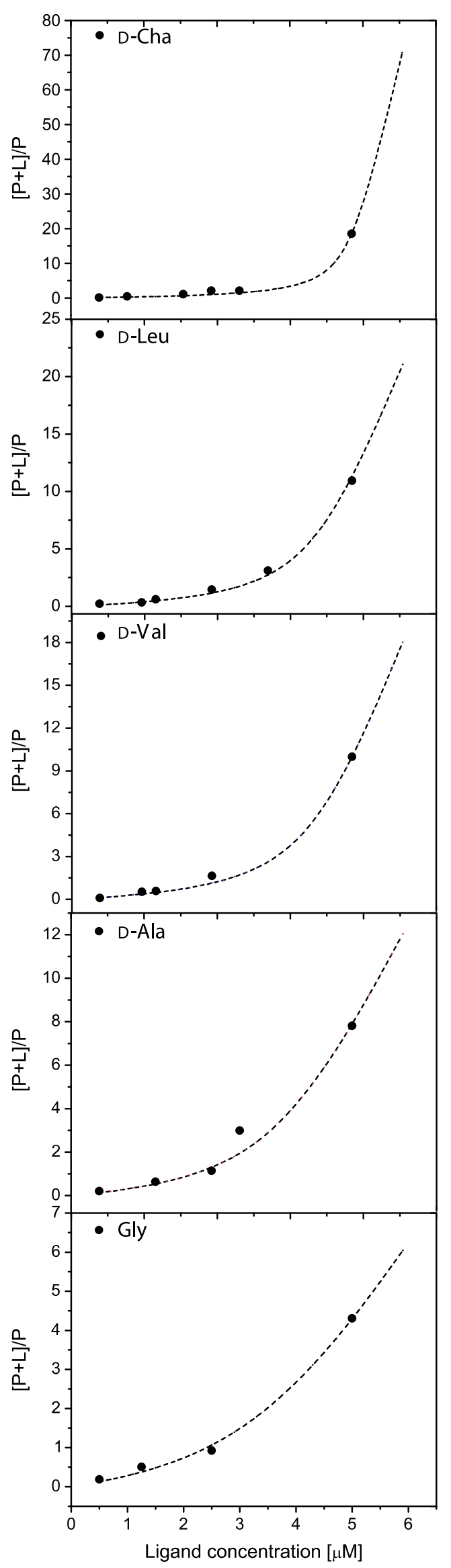


Table 2. List of Calculated Dissociation Constants $\left(\mathrm{K}_{\mathrm{D}}\right)$ for Five Benzamidine Based Ligands and Trypsin Determined by the NanoESI Titration Method, and $\mathrm{K}_{\mathrm{i}}$ Values from a Kinetic Inhibition Assay Determined in Solution for Trypsin and Thrombin

\begin{tabular}{cccc}
\hline Benzamidine series $(\mathrm{R})$ & \multicolumn{2}{c}{$\mathrm{K}_{\mathrm{D}}[\mathrm{nM}]$} & $\mathrm{K}_{\mathrm{i}}[\mathrm{nM}]$ \\
\cline { 2 - 4 } & nanoESI-MS trypsin $^{\mathrm{a}}$ & Kinetic inhibition assay trypsin $^{\mathrm{b}}$ & Kinetic inhibition assay thrombin $^{\mathrm{b}}$ \\
\hline D-Cha & $15.0 \pm 2.5$ & $0.95 \pm 0.18$ & $0.12 \pm 0.01$ \\
D-Leu & $79.3 \pm 5.9$ & $1.43 \pm 0.18$ & $0.89 \pm 0.12$ \\
D-Val & $87.1 \pm 15.2$ & $0.52 \pm 0.11$ & $1.29 \pm 0.35$ \\
D-Ala & $193.5 \pm 17.8$ & $1.95 \pm 0.77 ; 5 \pm 3^{\mathrm{c}}$ & $5.2 \pm 0.8$ \\
Gly & $449.7 \pm 75.9$ & $16 \pm 3 ; 37 \pm 3$ & $3.7 \pm 0.6$ \\
\hline
\end{tabular}

${ }^{a}$ Error is based on $95 \%$ confidence interval of the fitting curve

${ }^{\mathrm{b}}$ The error is given as the standard deviation calculated from at least three different measurements

'Second independent measurement

nate the noncovalent interaction, which is additionally modulated by a contribution from hydrophobic contacts.

\section{$K_{D}$-Determination of the CMA series by nanoESI-MS Titration Method}

The CMA-based inhibitors serve as a model system for weak binders in complex with trypsin. As shown in the spectra in Figure 6a, there is no change in the charge state distribution compared to the tighter binding benzamidine-based inhibitors. The $+7,+8,+9$ ions are predominantly observed in the spectrum. In Figure $6 \mathrm{a}-\mathrm{c}$ the mass spectra of $25 \mu \mathrm{M}$ Glyinhibitor/trypsin-complex was acquired using an accumula-

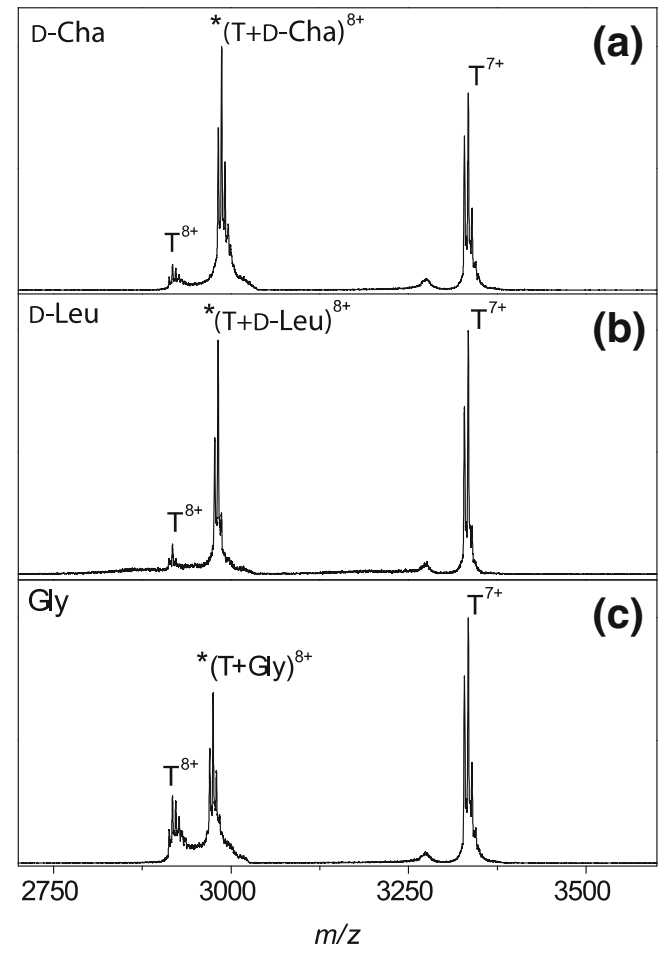

Figure 4. CID experiments of different noncovalent trypsin and benzamidine based inhibitors. For the MS/MS measurements the $8^{+}$-charged complex precursor ions $\left(^{*}\right)$ were selected. The collision energy was set to $20 \mathrm{~V}$. At the same collision energy, the D-Cha-complex stays $53 \%$ intact, for DLeu and Gly complexes $45 \%$, and $34 \%$ survive tion time of 30,60 , and $180 \mathrm{~s}$. We found that the relative abundance of protonated complex ions gradually decreased during acquisition of spectra. The longer accumulation time led to a decrease of $90 \%$ in the ratio of complex and bare protein, which would result in an artificially low binding affinity. The main reason of this observation is probably the in-source dissociation of the complex, as suggested previously by Klassen and co-workers [21]. One way to circumvent this limitation would be to use very short accumulation times. However, in order to achieve better signal-to-noise ratios in the mass spectrum, longer acquisition times are desirable. Even with the gentlest sampling conditions, the dissociation of the trypsin-CMA-inhibitor complexes could not be prevented. Therefore, other methods for stabilizing protein-ligand complexes were considered. It has recently been shown that ESI solution additives, such as imidazole, can protect the protein-ligand complex from dissociation.

The stabilization effect of imidazole in the presence of $25 \mu \mathrm{M}$ Gly-inhibitor is illustrated in Figure S1 (Supporting

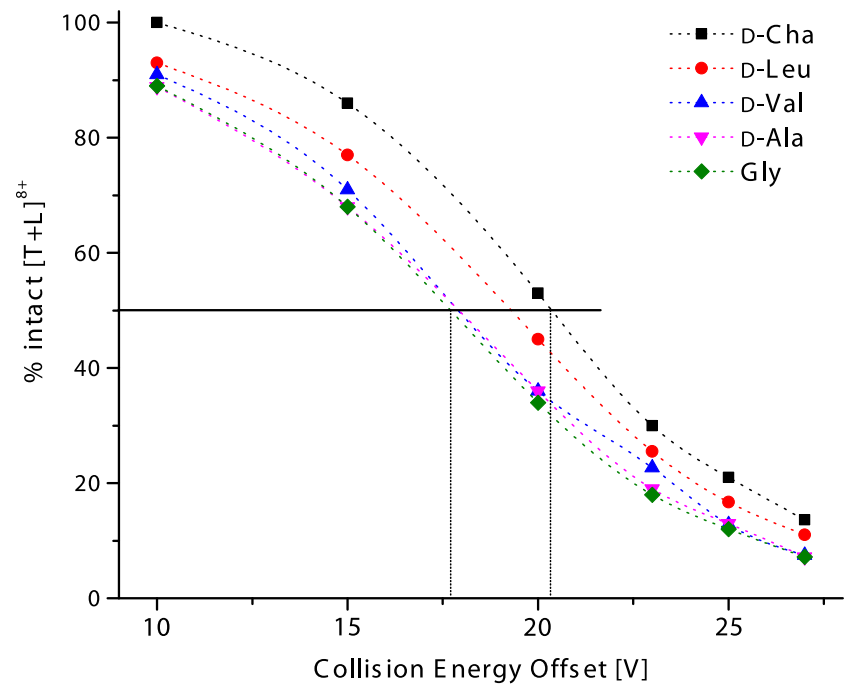

Figure 5. Normalized percentage of the intact complex plotted against the collision energy offset. A correlation between the gas phase stability and binding affinity is observed: With an increasing binding affinity, more energy is necessary to dissociate the complex 


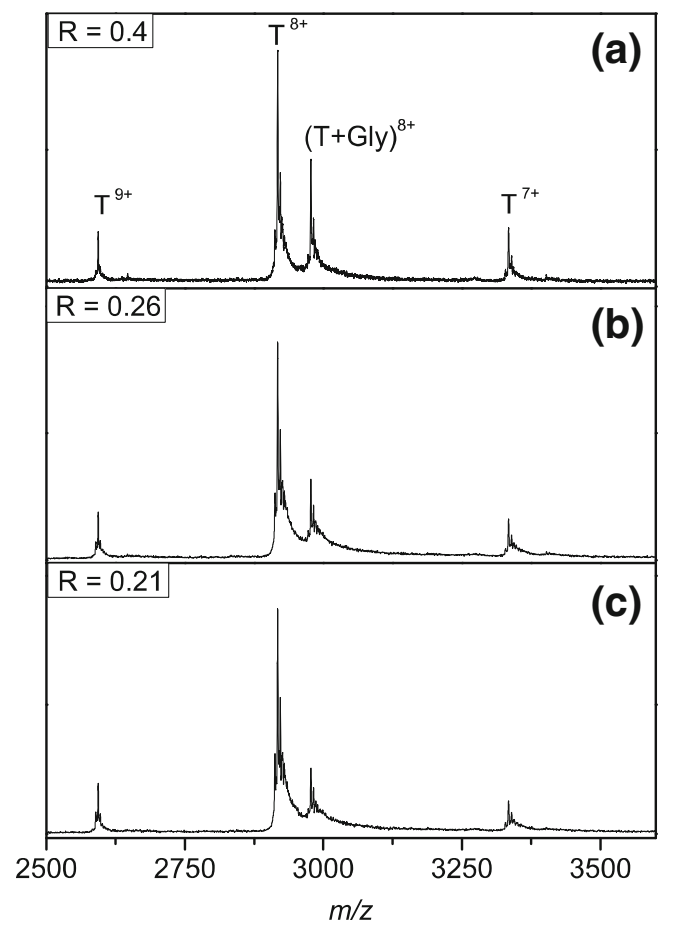

Figure 6. Representative nanoESI-MS spectra for $25 \mu \mathrm{M}$ Gly-inhibitor in complex with trypsin. The ratio $(R)$ between complex and bare protein decreases with increasing accumulation time after (a) $30 \mathrm{~s}(\mathrm{R}=0.4)$; (b) $60 \mathrm{~s}(\mathrm{R}=0.26)$; (c) 180 s $(R=0.21)$

Information). The nanoESI measurements were acquired under identical conditions as those in Figure 6 but now in the presence of $10 \mathrm{mM}$ imidazole in the ES-solution. Upon its addition to the nanoES solution, nonspecific adducts between the imidazole molecules and trypsin-ligand complexes can be formed. The nonspecific interactions should be kinetically less stable compared to specific trypsin-inhibitor complexes. After loss of the nonspecific interactions in the hexapole region, the internal energy of trypsin-ligand ions in the source should be lowered, thereby stabilizing the complex as shown in the spectra, the addition of imidazole to Gly-inhibitor/trypsin nanoES solution results in a double increase in the relative abundance of the complex ions. The important effect of this small molecule is the constant relative complex abundance measured over the longer accumulation time (data not shown). Also, the addition of imidazole reduces the average charge state distribution of the Gly-inhibitor/trypsin complex from $n=9-7$ to $n=8-6$ (Figure S1, Supporting Information). This stabilizing effect can be explained through enhanced cooling from imidazole evaporation that delays the dissociation. Coulomb repulsion can be minimized due to the reduced net charge state of the protein that stabilizes the complex [52]. Due to the high gas-phase basicity of imidazole $\left(217 \mathrm{kcal} \mathrm{mol}^{-1}\right)$, it is able to strip protons from the protonated protein ions in the gas phase [53]. As mentioned above, this observed charge stripping might play an important role in stabilizing of the complex, because the lower charge states are less susceptible to collisional dissociation. However, the appearance of the lower charge states is probably not the main mechanism for the complex stabilization, since $\mathrm{SF}_{6}$ provides similar complex stabilization without any shift of charge state distribution [27].

Another explanation for observing a drop in relative abundance of complex ions might be due to electrochemical reactions that occur where the electrode contacts the solution of the ES ion source. Products of such electrochemical reactions can alter the solution composition and affect the relative abundance of CMA complexes in solution and protonated complex ions during acquisition of spectra. As already shown [54] the solution composition and the resulting nanoESI spectra can be time dependent, with changes in the spectra being ascribed to on-going (electro)chemical reactions upstream in the capillary. It is conceivable that addition of imidazole can buffer a shift of the solution phase equilibrium, although the detailed mechanism of how this occurs is unknown.

This observation indicates that imidazole is a suitable additive for protecting the CMA-complexes during the nanoES process. Because the stabilization effect of imidazole on Gly-trypsin system appeared rather effective, we decided to perform further titration experiments with other CMA-inhibitors in the presence of imidazole. All titration experiments for CMA-complexes were carried out in positive ion mode. The only difference to the previous experiments is a higher concentration of the ligand $(1-30 \mu \mathrm{M})$, required to observe the weaker interactions and to obtain useful free protein-to-complex ratios.

The dissociation constants $\mathrm{K}_{\mathrm{D}}$ for all trypsin-inhibitor complexes were determined by fitting the five different titration curves as described above (data not shown). The results, given in Table 3, show higher dissociation constants compared to the benzamidine series, with $\mathrm{K}_{\mathrm{D}}$ values between 2.9 and $17.6 \mu \mathrm{M}$, some 1-2 orders of magnitude lower than the benzamidine complexes. The reason for lower

Table 3. List of Calculated Dissociation Constants $\left(\mathrm{K}_{\mathrm{D}}\right)$ for Five CMA Based Ligands Determined by the NanoESI Titration Method and Kinetic Inhibition Assay $\left(\mathrm{K}_{\mathrm{i}}\right)$ Determined in Solution. For Both Methods the Binding Affinity Increasing in Order Gly $<$ D-Ala $<$ D-Leu

\begin{tabular}{cccc}
\hline CMA series (R) & $\mathrm{K}_{\mathrm{D}}[\mu \mathrm{M}]$ & $\mathrm{K}_{\mathrm{i}}[\mu \mathrm{M}]$ & $\mathrm{K}_{\mathrm{i}}[\mathrm{nM}]$ \\
\cline { 2 - 4 } & $\begin{array}{l}\text { nanoESI-MS }_{\text {trypsin }^{\mathrm{a}}} \\
\text { Kinetic inhibition } \\
\text { assay trypsin }^{\mathrm{b}}\end{array}$ & $\begin{array}{l}\text { Kinetic inhibition } \\
\text { assay thrombin }\end{array}$ \\
\hline D-Cha & $17.63 \pm 1.2$ & $6.6 \pm 0.8$ & $0.052 \pm 0.005$ \\
D-Leu & $2.9 \pm 0.2$ & $0.4 \pm 0.09$ & $0.259 \pm 0.024$ \\
D-Val & $12.4 \pm 0.2$ & $6 \pm 0.2$ & $0.788 \pm 0.070$ \\
D-Ala & $8.4 \pm 1.4$ & $3.1 \pm 1$ & $2.2 \pm 0.4$ \\
Gly & $24.4 \pm 0.49$ & $9.9 \pm 0.1$ & $1.5 \pm 0.1$ \\
\hline
\end{tabular}

${ }^{a}$ Error is based on $95 \%$ confidence interval of the fitting curve

${ }^{\mathrm{b}}$ The error is given as the standard deviation calculated from at least three different measurements 
binding affinity compared to the previously described benzamidine based inhibitors is the substitution by the CMA anchor group, which is involved in the binding to the S1 pocket of trypsin. Moreover, unlike as for the benzamidine complexes, no trend in $\mathrm{K}_{\mathrm{D}}$ with increasing sidechain could be observed. However, an increased binding affinity is clearly observed in the order Gly $<$ D-Ala $<$ D-Leu (Table 3). Since the X-ray data for these complexes are not available, we assume that the different trend of binding affinities is probably due to the different anchor groups of the different ligand series.

The relative difference in the $\mathrm{K}_{\mathrm{D}}$ values determined by nanoESI-MS and the characterized $K_{i}$ values are equivalent, considering the error margins of the methods. These results confirm the same trend observed in the kinetic inhibition assay as under the experimental MS conditions, demonstrating the ability of quantitative ESIMS measurement to clearly distinguish between ligand affinities.

\section{Conclusions}

In conclusion, we have applied nanoESI-MS for the investigation of a series of hydrophobically modified ligands interacting with trypsin. The size of the hydrophobic side chain $(\mathrm{R})$ that binds in the $\mathrm{S} 3 / 4$ pocket of trypsin was systematically increased. The different substituents $(\mathrm{R}=$ Gly, D-Ala, D-Val, D-Leu, D-Cha) have a significant influence on the binding constants. The quantification of binding affinities was possible using the titration method. In the case of the benzamidine series the trend to higher binding affinity with increasing hydrophobic P3 side chain is strong, ranging from $450 \mathrm{nM}$ to $15 \mathrm{nM}$. The binding affinities measured by ESI-MS titration and kinetic inhibition constants for the benzamidine-trypsin complexes show, with the exception of D-Val, the same relative ordering. Collision-induced dissociation experiments across the benzamidene type series clearly show the correlation between the binding affinity and the gas phase stability. More collision energy is necessary to dissociate the complex with higher binding affinity and vice versa.

The CMA-inhibitors served as a model system for a series of less potent complexes, which are prone to insource dissociation. This effect causes a reduced relative abundance of the gaseous complex ions and leads therefore to artificially lower binding affinities in the measurements. Upon addition of imidazole, a stabilizing solution additive, the relative abundance of the nondissociated could be increased. Compared to benzamidine-inhibitors the CMA ligands did not show the clear trend towards higher binding strengths with longer sidechains. The increased binding affinity is observed for Gly $<$ D-Ala $<$ D-Leu.

For the CMA series the relative difference for $\mathrm{K}_{\mathrm{D}}$ and the characterized $K_{i}$ values are equivalent, which demonstrates the ability of quantitative ESI-MS to distinguish between ligand affinities.

\section{Acknowledgments}

The authors acknowledge support for this work by the CHEBANA-Project (grant no. 264772).

\section{References}

1. Hofstadler, S.A., Sannes-Lowery, K.A.: Applications of ESI-MS in drug discovery: Interrogation of noncovalent complexes. Nat. Rev. Drug Discov. 5(7), 585-595 (2006)

2. Daniel, J.M., Friess, S.D., Rajagopalan, S., Wendt, S., Zenobi, R.: Quantitative determination of noncovalent binding interactions using soft ionization mass spectrometry. Int. J. Mass Spectrom. 216, 1-27 (2002)

3. Tjernberg, A., Carno, S., Oliv, F., Benkestock, K., Edlund, P.O., Griffiths, W.J., Hallen, D.: Determination of dissociation constants for protein-ligand complexes by electrospray ionization mass spectrometry. Anal. Chem. 76, 4325-4331 (2004)

4. Wortmann, A., Jecklin, M.C., Touboul, D., Badertscher, M., Zenobi, R.: Binding constant determination of high-affinity protein-ligand complexes by electrospray ionization mass spectrometry and ligand competition. J. Mass Spectrom. 43, 600-608 (2007)

5. Zhang, J., McCombie, G., Guenat, C., Knochenmuss, R.: FT-ICR mass spectrometry in the drug discovery process. Drug Discov. Today $\mathbf{1 0}$, 635-642 (2005)

6. Deng, G.J., Sanyal, G.: Applications of mass spectrometry in early stages of target based drug discovery. J. Pharm. Biomed. Anal. 40, 528538 (2006)

7. Loo, J.A.: Studying noncovalent protein complexes by electrospray ionization mass spectrometry. Mass Spectrom. Rev. 16, 1-23 (1997)

8. Van den Heuvel, R.H., Heck, A.J.: Native protein mass spectrometry: From intact oligomers to functional machineries. Curr. Opin. Chem. Biol. 8, 519-526 (2004)

9. Zhu, M.M., Rempel, D.L., Du, Z., Gross, M.L.: Quantification of protein-ligand interactions by mass spectrometry, titration, and H/D exchange: PLIMSTEX. J. Am. Chem. Soc. 125, 5252-5253 (2003)

10. Zhu, M.M., Chitta, R., Gross, M.L.: A novel mass spectrometric method for the quantification of protein-ligand interactions in solution. Int. J. Mass Spectrom 240, 213-240 (2005)

11. Ghaemmaghami, S., Fitzgerald, M.C., Oas, T.G.: A quantitative, highthroughput screen for protein stability. Proc. Natl. Acad. Sci. U.S.A. 97, 8296-8301 (2000)

12. Southall, N.T., Dill, K.A., Haymet, A.D.J.: A view of the hydrophobic effect. J. Phys. Chem. B 106(10), 2812-2812 (2002)

13. Pace, C., Shirley, B., McNutt, M., Gajiwala, K.: Forces contributing to the conformational stability of proteins. FASEB J. 10(1), 75-83 (1996)

14. Ball, P.: Water as an active constituent in cell biology. Chem. Rev. 108 (1), 74-108 (2008)

15. Kauzmann, W.: Some factors in the interpretation of protein denaturation. Adv. Protein Chem. 14, 1-63 (1959)

16. Jørgensen, T.J.D., Delforge, D., Remacle, J., Bojesen, G., Roepstorff, P.: Collision-induced dissociation of noncovalent complexes between vancomycin antibiotics and peptide ligand stereoisomers: Evidence for molecular recognition in the gas phase. Int. J. Mass Spectrom. 188, 6385 (1999)

17. Wu, Q.Y.: Carbonic anhydrase-inhibitor binding: From solution to the gas phase. J. Am. Chem. Soc. 119, 1157-1158 (1997)

18. Veros, C.T., Oldham, N.J.: Quantitative determination of lysozymeligand binding in the solution and gas phases by electrospray ionisation mass spectrometry. Rapid Commun. Mass Spectrom. 21(21), 35053510 (2007)

19. Frański, R., Gierczyk, B., Schroeder, G., Frańska, M., Wyrwas, B.: Do hydrophobic interactions exist in the gas phase? Rapid Commun. Mass Spectrom. 22(8), 1339-1343 (2008)

20. Barylyuk, K., Balabin, R., Grönstein, D., Kikkeri, R., Frankevich, V., Seeberger, P., Zenobi, R.: What happens to hydrophobic interactions during transfer from the solution to the gas phase? The case of electrospray-based soft ionization methods. J. Am. Soc. Mass Spectrom. 22(7), 1167-1177 (2011) 
21. Liu, L., Kitova, E., Klassen, J.: Quantifying protein-fatty acid interactions using electrospray ionization mass spectrometry. $J$. Am. Soc. Mass Spectrom. 22(2), 310-318 (2011)

22. El-Hawiet, A., Kitova, E., Liu, L., Klassen, J.: Quantifying labile protein-ligand interactions using electrospray ionization mass spectrometry. J. Am. Soc. Mass Spectrom. 21(11), 1893-1899 (2010)

23. Liu, L., Bagal, D., Kitova, E.N., Schnier, P.D., Klassen, J.S.: Hydrophobic protein-ligand interactions preserved in the gas phase. $J$. Am. Chem. Soc. 131(44), 15980-15981 (2009)

24. Liu, L., Michelsen, K., Kitova, E.N., Schnier, P.D., Klassen, J.S.: Evidence that water can reduce the kinetic stability of proteinhydrophobic ligand interactions. J. Am. Chem. Soc. 132(50), 1765817660 (2010)

25. Bich, C., Baer, S., Jecklin, M., Zenobi, R.: Probing the hydrophobic effect of noncovalent complexes by mass spectrometry. J. Am. Soc. Mass Spectrom. 21(2), 286-289 (2010)

26. Sun, J.X., Kitova, E.N., Klassen, J.S.: Method for stabilizing proteinligand complexes in nanoelectrospray ionization mass spectrometry. Anal. Chem. 79(2), 416-425 (2007)

27. Bagal, D., Kitova, E.N., Liu, L., El-Hawiet, A., Schnier, P.D., Klassen, J.S.: Gas phase stabilization of noncovalent protein complexes formed by electrospray ionization. Anal. Chem. 81(18), 7801-7806 (2009)

28. Robinson, C.V.: Probing the nature of noncovalent interactions by mass spectrometry. A study of protein-coA ligand binding and assembly. $J$. Am. Chem. Soc. 118, 8646-8653 (1996)

29. Clark, S.M., Konermann, L.: Determination of ligand-protein dissociation constants by electrospray mass spectrometry-based diffusion measurements. Anal. Chem. 76(23), 7077-7083 (2004)

30. Bovet, C., Wortmann, A., Eiler, S., Granger, F., Ruff, M., Gerrits, B., Moras, D., Zenobi, R.: Estrogen receptor-ligand complexes measured by chip-based nanoelectrospray mass spectrometry: an approach for the screening of endocrine disruptors. Protein Sci. 16(5), 938-946 (2007)

31. Jecklin, M., Touboul, D., Bovet, C., Wortmann, A., Zenobi, R.: Which electrospray-based ionization method best reflects protein-ligand interactions found in solution? A comparison of ESI, nanoESI, and ESSI for the determination of dissociation constants with mass spectrometry. $J$. Am. Soc. Mass Spectrom. 19(3), 332-343 (2008)

32. Jecklin, M.C., Schauer, S., Dumelin, C.E., Zenobi, R.: Label-free determination of protein-ligand binding constants using mass spectrometry and validation using surface plasmon resonance and isothermal titration calorimetry. J. Mol. Rec. 22(4), 319-329 (2009)

33. Jørgensen, T.J.D., Roepstorff, P.: Direct determination of solution binding constants for noncovalent complexes between bacterial cell wall peptide analogues and vancomycin group antibiotics by electrospray ionization mass spectrometry. Anal. Chem. 70, 4427-4432 (1998)

34. Hagan, N., Fabris, D.: Direct mass spectrometric determination of the stoichiometry and binding affinity of the complexes between nucleocapsid protein and RNA stem-loop hairpins of the HIV-1 Psi-recognition element. Biochemistry 42, 10736-10745 (2003)

35. Talhout, R., Engberts, J.B.F.N.: Thermodynamic analysis of binding of p-substituted benzamidines to trypsin. Eur. J. Biochem. 268, 1554-1560 (2001)

36. Wang, Y., Luo, W., Reiser, G.: Trypsin and trypsin-like proteases in the brain: Proteolysis and cellular functions. Cell. Mol. Life Sci. 65(2), 237$252(2008)$

37. Rogniaux, H.: Binding of aldose reductase inhibitors: Correlation of crystallographic and mass spectrometric studies. J. Am. Soc. Mass Spectrom. 10, 635-647 (1999)
38. Cheng, X.: Using electrospray ionization FTICR mass spectrometry to study competitive binding of inhibitors to carbonic anhydrase. $J$. Am. Chem. Soc. 117, 8859-8860 (1995)

39. Potier, N., Donald, L.J., Chernushevich, I., Ayed, A., Ens, W., Arrowsmith, C.H., Standing, K.G., Duckworth, H.W.: Study of a noncovalent trp repressor: DNA operator complex by electrospray ionization time-of-flight mass spectrometry. Protein Sci. 7, 1388-1395 (1998)

40. Yin, S., Xie, Y., Loo, J.A.: Mass spectrometry of protein-ligand complexes: enhanced gas-phase stability of ribonuclease-nucleotide complexes. J. Am. Soc. Mass Spectrom. 19, 1199-1208 (2008)

41. Li, Y.T., Hsieh, Y.L., Henion, J.D., Senko, M.W., McLafferty, F.W., Ganem, B.: Mass-spectrometric studies on noncovalent dimers of leucine-zipper peptides. J. Amer. Chem. Soc. 115(18), 8409-8413 (1993)

42. Little, D.P., Speir, J.P., Senko, M.W., O'Connor, P.B., McLafferty, F.W.: Infrared multiphoton dissociation of large multiply charged ions for biomolecule sequencing. Anal. Chem. 66, 2809-2815 (1994)

43. Wendt, H., Durr, E., Thomas, R.M., Przybylski, M., Bosshard, H.R.: Characterization of leucine zipper complexes by electrospray ionization mass spectrometry. Protein Sci. 4, 1563-1570 (1995)

44. Nesatyy, V.J.: Gas-phase binding of non-covalent protein complexes between bovine pancreatic trypsin inhibitor and its target enzymes studied by electrospray ionization tandem mass spectrometry. J. Mass Spectrom. 36, 950-959 (2001)

45. Jecklin, M.C., Touboul, D., Jain, R., Toole, E.N., Tallarico, J., Drueckes, P., Ramage, P., Zenobi, R.: Affinity classification of kinase inhibitors by mass spectrometric methods and validation using standard $\mathrm{IC}_{50}$ measurements. Anal. Chem. 81(1), 408-419 (2008)

46. Sharon, M., Robinson, C.V.: A quantitative perspective on hydrophobic interactions in the gas-phase. Curr. Proteom. 8(1), 47-58 (2011)

47. Stürzebecher, J., Stürzebecher, U., Vieweg, H., Wagner, G., Hauptmann, J., Markwardt, F.: Synthetic inhibitors of bovine factor $\mathrm{Xa}$ and thrombin comparison of their anticoagulant efficiency. Thromb. Res. 54(3), 245-252 (1989)

48. Ota, N., Stroupe, C., Ferreira-da-Silva, J.M.S., Shah, S.A., Mares-Guia, M., Brunger, A.T.: Non-Boltzmann thermodynamic integration (NBTI) for macromolecular systems: Relative free energy of binding of trypsin to benzamidine and benzylamine. Proteins 37(4), 641-653 (1999)

49. Peters, L., Frohlich, R., Boyd, A.S.F., Kraft, A.: Noncovalent interactions between tetrazole and an $N, N^{\prime}$-diethyl-substituted benzamidine. J. Org. Chem. 66, 3291-3298 (2001)

50. Brandt, T., Holzmann, N., Muley, L., Khayat, M., Wegscheid-Gerlach, C., Baum, B., Heine, A., Hangauer, D., Klebe, G.: Congeneric but still distinct: How closely related trypsin ligands exhibit different thermodynamic and structural properties. J. Mol. Biol. 405(5), 1170-1187 (2011)

51. Di Fenza, A., Heine, A., Koert, U., Klebe, G.: Understanding binding selectivity toward trypsin and factor Xa: the role of aromatic interactions. ChemMedChem 2(3), 297-308 (2007)

52. Lorenzen, K., Duijn, E.V.: Native mass spectrometry as a tool in structural biology. Curr. Protoc. Protein Sci. 62, 17.12.1-17.12.17 (2010)

53. Hunter, E.P.L., Lias, S.G.: Evaluated gas phase basicities and proton affinities of molecules: An update. J. Phys. Chem. Ref. Data 27(3), 413-656 (1998)

54. Van Berkel, G.J., Asano, K.G., Schnier, P.D.: Electrochemical processes in a wire-in-a-capillary bulk-loaded, nano-electrospray emitter. J. Am. Soc. Mass Spectrom. 12(7), 853-862 (2001) 